\title{
HASHIMOTO'S ENCEPHALOPATHY WITH CLINICAL FEATURES SIMILAR TO THOSE OF CREUTZFELDT-JAKOB DISEASE
}

\author{
Ana Cláudia Rodrigues de Cerqueira ${ }^{1,2 A}$, José Marcelo Ferreira Bezerra ${ }^{7 B}$, \\ Gérson Canedo de Magalhães ${ }^{1 C}$, Márcia Rozenthal ${ }^{3 B}$, Antônio Egídio Nardii ${ }^{2 B}$
}

In 1966, Brain et al. described the first case of Hashimoto's encephalopathy in a patient with stroke-like episodes, seizure activities, and delirium. The symptoms were recurrent and reversible and were not associated with thyroid dysfunction'. Since then, Hashimoto's encephalopathy has been recognized as a rare condition associated to Hashimoto's thyroiditis and to the presence of high concentrations of antithyroid antibodies, unlike those described in myxedema and in thyrotoxicosis ${ }^{2}$. Two clinical forms have been described: the vasculitic form with stroke-like episodes, seizure activities, and slight cognitive decline; and the diffuse form with symptoms of depression, psychosis, myoclonus, tremors, delusion, fluctuations in the level of consciousness, and dementia. The clinical manifestations can be fluctuating or persistent, and reversible, if properly treated with immunosuppressive therapy ${ }^{3}$. In some cases, Hashimoto's encephalopathy can show rapidly progressive dementia, myoclonus, and electroencephalographic changes with periodic recurrence of the triphasic complexes similar to those of Creutzfeldt-Jakob disease, which makes a differential diagnosis essential to both pathologies ${ }^{4}$.

We describe the case of a patient with Hashimoto's encephalopathy who showed clinical manifestations similar to those of Creutzfeldt-Jakob disease. The recognition of the condition was essential for the treatment and regression of the clinical presentation.

\section{CASE}

A female patient, 68-years-old, Caucasian, widow, born in Rio de Janeiro, developed a clinical presentation of daily sadness, discouragement, decreased appetite, insomnia, and cognitive decline. The condition of the patient worsened with persecutory delusions, visual hallucinations, gait disturbance, tremor of the extremities, muscle spasms, and fluctuations in the level of consciousness. She had a history of hypothyroidism and did not use any medication. At admission, she was sleepy, disoriented, showing myoclonus, paratonia on the back of the head and arms, her deep reflexes were symmetrical with plantar flexion response and suction and grasping reflexes were present. The rest of the physical examination was normal.

The laboratory exams, including a complete blood count; biochemistry; arterial gasometry; hepatic function tests; autoantibody tests, such as anti-nuclear factor (ANF), anti-Ro, antiLa, pANCA and cANCA, were either normal or negative. The lev-

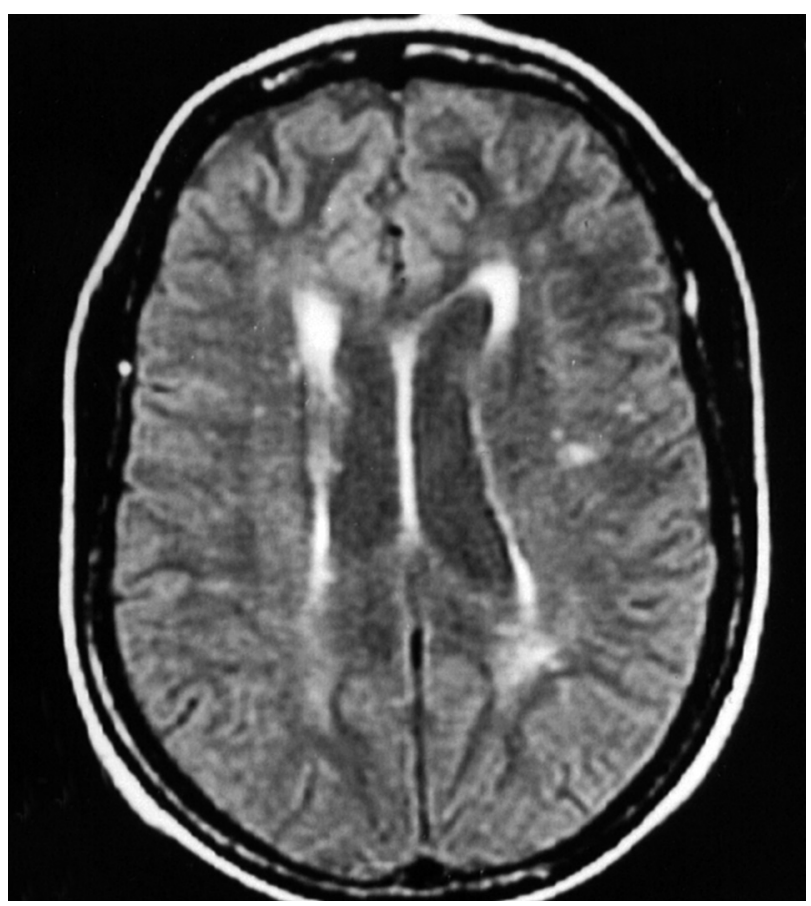

Fig 1. MRI of the brain, axial section of a patient diagnosed with Hashimoto's encephalopathy.

\section{ENCEFALOPATIA DE HASHIMOTO COM CARACTERÍSTICAS CLÍNICAS SEMELHANTES À DOENÇA DE CREUTZFELDT-JAKOB}

Pedro Ernesto University Hospital of the University of the State of Rio de Janeiro, Rio de Janeiro RJ, Brazil (HUPE/UERJ): ${ }^{1}$ Neurology Service; ${ }^{2}$ Institute of Psychiatry at the Federal University of Rio de Janeiro, Rio de Janeiro RJ, Brazil (IPUB/UFRJ); ${ }^{3}$ Federal University of the State of Rio de Janeiro, Rio de

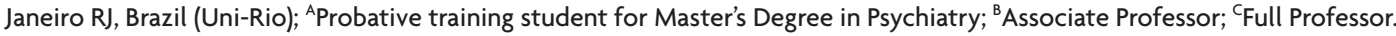

Received 18 June 2008, received in final form 1 September 2008. Accepted 23 September 2008.

Dra. Ana Claudia Rodrigues de Cerqueira - Rua Trajano de Moraes 42 /701 - 24230-380 Niterói RJ - Brasil. E-mail: anacerqueira@globo.com 


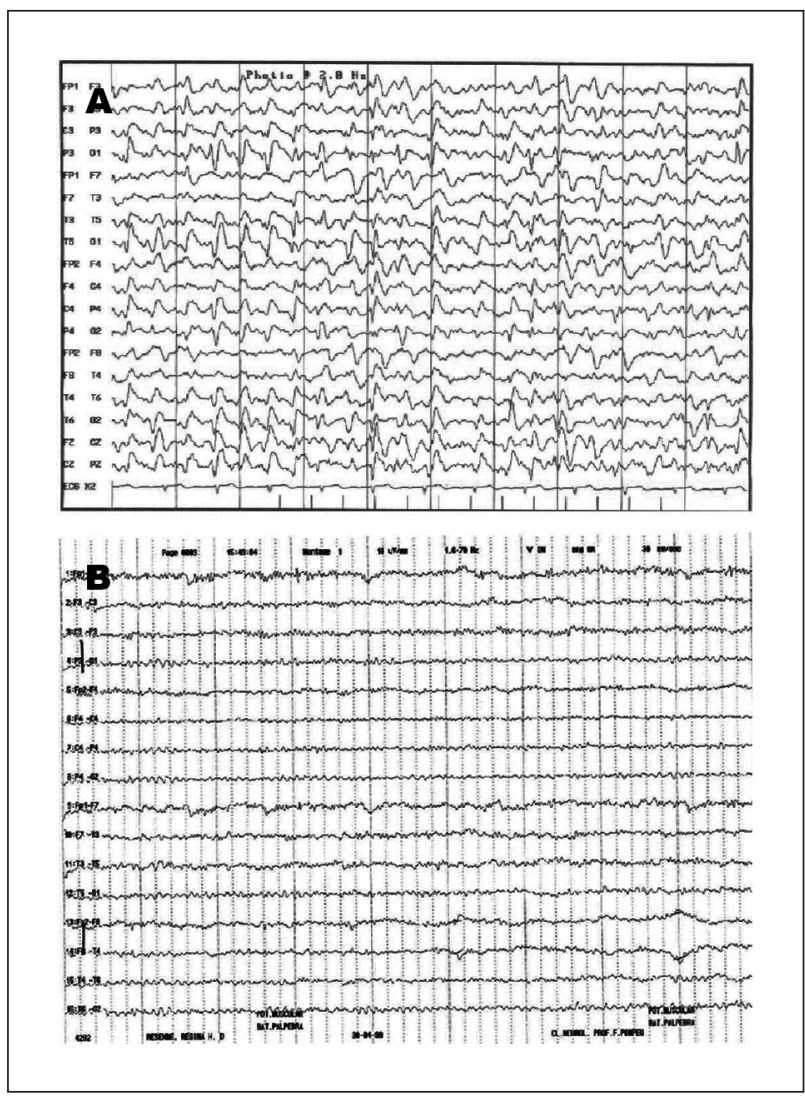

Fig 2. EEG of a 68-year-old the patient diagnosed with Hashimoto's encephalopathy. (A) At admission-presence of triphasic complexes. (B) After 2 months of treatment- EEG under normal variation values.

els of triiodothyronine (T3) and free thyroxine (T4 L) were within normal limits. The thyroid-stimulating hormone (TSH) was slightly elevated at $7.70 \mathrm{mU} / \mathrm{L}$ (reference value: $0.35-5.5 \mathrm{mU} / \mathrm{L}$ ). The anti-thyroperoxidase (anti-TPO) concentration was very high at 1/6400 (reference value: up to 1/100). The presence of antithyroglobulin antibody (Ac-anti-Tg) was not detected. The examination of the cerebrospinal fluid (CSF), including cytometry, biochemistry, immunoglobulins, detection of oligoclonal bands and testing for the presence of bacteria and fungi, immunological reactions for syphilis, cysticercosis, schistosomiasis, toxoplasmosis, cytomegalovirus, HIV, HTLV1/2, herpes simplex and herpes zoster were either normal or negative.

The magnetic resonance imaging (MRI) of the brain showed small changes that were not significant, such as the presence of hypersignal focus in the corona radiata and bilaterally semioval center (Fig 1). The electroencephalogram (EEG) at admission was abnormal, with slow, monomorphic, occasionally sharp, triphasic, with theta-delta frequency ranges from 2 to $3 \mathrm{~Hz}$, intermittent, bilateral waves, predominantly in the temporoparietal regions (Fig 2A).

The diagnosis hypothesized was of Hashimoto's encephalopathy and the treatment with methylprednisolone $1 \mathrm{~g} /$ day for three consecutive days, followed by prednisone with initial dose of $60 \mathrm{mg} /$ day, was established. In the first two weeks, a considerable improvement in level of consciousness and myoclonus was observed. The prednisone dosage was gradually reduced in the following two months to $5 \mathrm{mg} /$ day. In this period there was a regression of all signs and symptoms. This improvement in the clinical presentation can be correlated to the complete resolution of the electroencephalographic changes (Fig 2B). The patient authorized the publication of the case upon signature of an informed consent form.

\section{DISCUSSION}

Hashimoto's encephalopathy is a rare condition associated with Hashimoto thyroiditis, the presence of high concentrations of anti-thyroid antibodies, without any evidence of thyroid dysfunction, and an excellent response to treatment with corticosteroids ${ }^{2}$. Recent studies have shown the presence of anti-thyroid antibodies in the $\mathrm{CSF}^{5}$ and alpha-enolase antibodies in the serum of patients with Hashimoto's encephalopathy, suggesting the involvement of an autoimmune mechanism in this condition ${ }^{6}$. However, the role of those antibodies and their pathophysiology are unknown. A possible vasculitic process has been shown in biopsy studies ${ }^{7}$.

The clinical presentation may suggest a diagnosis of Creutzfeldt-Jakob disease. In an epidemiological study made by Seipelt et al., six cases of Hashimoto's encephalopathy were identified and treated. The patients met the clinical criteria for Creutzfeldt-Jakob disease and were notified $^{8}$. The criteria established by World Health Organization (WHO) for diagnosing to sporadic Creutzfeldt-Jakob disease (sCJD) include progressive dementia with a duration of less than two years and two of the clinical findings - myoclonus, cerebellar or visual symptoms, pyramidal or extrapyramidal signs and akinetic mutism. The clinical manifestations associated with the presence of triphasic complexes in the EEG or the detection of the 14-3-3 protein in the CSF make the diagnosis possible. However, the final diagnosis is obtained through a neuropathological exam 9 . A recent study by Martins et al. described the first 35 cases of Creutzfeldt-Jakob disease notified in Brazil, 26 of these cases were initially classified as possible sCJD, of which $51 \%$ fulfilled the criteria for probable sCJD. The most frequent clinical manifestations were myoclonus (80\%), pyramidal signs (68\%), ataxia (65\%) and early psychiatric symptoms $(55 \%)^{10}$.

We related the case of a patient with Hashimoto's encephalopathy and clinical features similar to those of Creutzfeldt-Jakob disease. The presence rapidly progressive dementia, ataxia, myoclonus, and especially the presence of triphasic complexes in the EEG, make the diagnosis a probable one for Creutzfeldt-Jakob disease. In this case, the Hashimoto's encephalopathy diagnosis was 
made based on the exclusion of other toxic-metabolic encephalopathic and neurological diseases of infectious or vascular origin, and on the detection of high concentrations of anti-TPO antibodies. The recognition of this pathology and the treatment with immunosuppressant therapy determined the regression of the clinical presentation and changes in the EEG.

This report emphasizes the importance of a differential diagnosis between the two pathologies. CreutzfeldtJakob disease has a progressive and inexorably fatal course, as opposed to Hashimoto's encephalopathy, which has a fluctuating course and excellent response to treatment with corticosteroids. We believe that this pathology is misdiagnosed, therefore, tests detecting the presence of anti-thyroid antibodies are recommended in a clinical presentation of encephalopathy of subacute onset or rapidly progressive dementia associated with myoclonus and triphasic complexes in the EEG, when the ethiology is not identified.

\section{REFERENCES}

1. Brain L, Jellinek EH, Ball K. Hashimoto's disease and encephalopathy. Lancet 1966;2:512-514.

2. Chong JY, Rowland LP, Utiger RD. Hashimoto encephalopathy: syndrome or myth? Arch Neurol 2003;60:164-171.

3. Kothbauer-Margreiter I, Sturzenegger M, Komor J, Baumgartner R, Hess C W. Encephalopathy associated with Hashimoto thyroiditis: diagnosis and treatment. J Neurol 1996;243:585-593.

4. Doherty CP, Schlossmacher M, Torres N, Bromfield E, Samuels MA Hashimoto's encephalopathy mimicking Creutzfeldt-Jakob disease: brain biopsy findings. J Neurol Neurosurg Psychiatry 2002;73:597-603.

5. Ferraci F,Moretto G, Candeago RM et al. Antithyroid antibodies in the CSF: their role in the pathogenesis of Hashimoto's encefalopathy. Neurology 2003;60:712-714.

6. Ochi H, Horiuchi I, Araki N, et al. Proteomic analysis of human brain identifies alpha-enolase as a novel autoantigen in Hashimoto's encephalopathy. FEBS Lett 2002;528:197-202.

7. Shibata N, Yamamoto Y, Sunami N, Suga M, Yamashita Y. Isolated angiitis of the CNS associated with Hashimoto's disease. Rinsho Shinkeigaku 1992;32:191-198.

8. Seipelt M, Zerr I, Nau R, et al. Hashimoto's encephalitis as a differential diagnosis of Creutzfeldt-Jakob disease. J Neurol Neurosurg Psychiatry 1999;53:162-163.

9. WHO (see http://www.who.int/entity/zoonoses/diseases/ Creutzfeldt.pdf)

10. Martins VR, Gomes HR, Chimelli L, Rosemberg S, Landemberger MC. Prion diseases are under compulsory notification in Brazil: surveillance of cases evaluated by biochemical and / or genetic markers from 2005 to 2007. Dementia \& Neuropsychol 2007;1:347-355. 